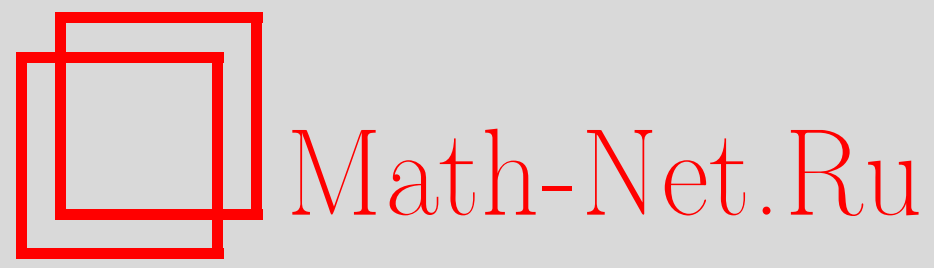

Л. И. Сербина, Решение одной начально-краевой задачи теории фильтрации с нелокальными краевыми условиями, Вестн. Сам. гос. техн. ун-та. Сер. Физ.-мат. науки, 2003, выпуск 19, 16-21

DOI: https://doi.org/10.14498/vsgtu133

Использование Общероссийского математического портала Math-Net.Ru подразумевает, что вы прочитали и согласны с пользовательским соглашением

http: //www.mathnet.ru/rus/agreement

Параметры загрузки:

IP : 54.237 .59 .107

26 апреля 2023 г., $16: 16: 51$ 


\section{Л.И. Сербина}

\section{РЕШЕНИЕ ОДНОЙ НАЧАЛЬНО-КРАЕВОЙ ЗАДАЧИ ТЕОРИИ ФИЛЬТРАЦИИ \\ С НЕЛОКАЛЬНЫМИ КРАЕВЫМИ УСЛОВИЯМИ}

Приведено решение начально - краевой задачи фильтращии неустановившегося одномерного движения грунтовых вод, описываемой уравнением Буссинеска. Исследованы различные частные случаи основного уравнения, в частности сводящиеся к уравнению Лаврентьева - Бицадзе с нелокальными краевыми условиями. Сформулирован ряд краевых задач и построены их решения.

Аппроксимация обобщенного нелинейного уравнения Буссинеска. В классической теории фильтрации неустановившееся одномерное движение грунтовых вод со слабоизменяющейся свободной поверхностью и с горизонтальным водоупором описывается обобщенным уравнением Буссинеска:

$$
\sigma \frac{\partial h}{\partial t}=k \frac{\partial}{\partial \xi}\left[h \frac{\partial h}{\partial \xi}\right]-\frac{k_{0}}{M_{0}}\left(h-H_{0}\right)+w,
$$

где постоянные $\sigma$ и $\quad k$ означают водоотдачу и коэффициент фильтрации; $h=h(\xi, t)$ - уровень грунтовой воды в точке $\xi \geq 0$ в момент времени $t$; $k_{0}$ - коэффициент фильтрации слабопроницаемого водоупора мощности $M_{0} ; w=w(\xi, t)$ - разность между инфильтрацией и испарением; $H_{0}$ - напор воды в нижележащем водоносном пласте. Любое регулярное решение уравнения (1) удовлетворяет уравнению

$$
\sigma \frac{\partial^{2} h}{\partial t^{2}}=k \frac{\partial^{2}}{\partial \xi^{2}}\left[h \frac{\partial h}{\partial t}\right]-\frac{k_{0}}{M_{0}} \frac{\partial h}{\partial t}+\frac{\partial w}{\partial t} .
$$

Предположим, что $h_{t}=\partial h / \partial t$ в среднем прямо пропорционально расходу грунтовой воды на прогнозируемом участке $0<\xi<l$ с постоянным коэффициентом $\gamma$ :

$$
\frac{\partial h}{\partial t} \approx \gamma \sigma \frac{\partial}{\partial t} \int_{0}^{l} h(\xi, t) d \xi
$$

Гипотеза (3) позволяет за приближенное решение уравнения (2) принять точное решение следующего уравнения:

$$
\frac{\partial^{2} h}{\partial t^{2}}=k \gamma \delta \delta^{\prime}(t) \frac{\partial^{2} h}{\partial \xi^{2}}-\frac{1}{\sigma}\left(\frac{k_{0}}{M_{0}} \frac{\partial h}{\partial t}-\frac{\partial w}{\partial t}\right)
$$

где $\delta(t)=\int_{0}^{l} h(\xi, t) d \xi$.

Для широкого класса мелиоративных задач прогнозирования динамики грунтовых вод можно положить, что

$$
k \gamma \delta^{\prime}(t) \approx c\left|t_{*}-t\right|^{m} \operatorname{sign}\left(t_{*}-t\right),
$$

где $m=$ const $>0, c=$ const $>0, t_{*}-$ экстремальное время, когда расход грунтовой воды в слое $0<\xi<l$ достигает максимального значения, а затем падает до значения, не нарушающего экологию зоны аэрации.

Из (4) в силу (5) получим уравнение

$$
\frac{\partial^{2} h}{\partial t^{2}}=c\left|t_{*}-t\right|^{m} \operatorname{sign}\left(t_{*}-t\right) \frac{\partial^{2} h}{\partial \xi^{2}}-\frac{k_{0}}{\sigma M_{0}} \frac{\partial h}{\partial t}+\frac{1}{\sigma} \frac{\partial w}{\partial t} .
$$

Уравнение (6) на евклидовой плоскости точек $(\xi, t)$ является уравнением смешанного типа: оно эллиптично при $t>t_{*}$, гиперболично при $t<t_{*}$ и параболично на критической линии $t_{*}=0$.

Заменой переменных

$$
y=t-t_{*}, x=\xi / \sqrt{c}, u(x, y)=h\left(x \sqrt{c}, y+t_{*}\right)
$$


уравнение (6) приведем к виду

$$
\frac{\partial^{2} u}{\partial y^{2}}+\operatorname{sign} y|y|^{m} \cdot \frac{\partial^{2} u}{\partial x^{2}}+b \frac{\partial u}{\partial y}=f(x, y),
$$

где $b=\frac{k_{0}}{\sigma \cdot M_{0}}, f(x, y)=\frac{1}{\sigma} \frac{\partial}{\partial y} w\left(x \sqrt{c}, y+t_{*}\right)$.

Для уравнения (8) на основании (3) и (5) получим нелокальное условие

$$
\frac{\partial}{\partial y} \int_{0}^{r} u(x, y) d x=A|y|^{m} \operatorname{sign} y .
$$

Здесь использованы обозначения $r=l / \sqrt{c}, A=-\sqrt{c} / k \gamma$.

Итак, уравнение (8) смешанного эллиптико-гиперболического типа является линейной математической моделью динамики грунтовых вод, адекватно отражающей явление конечности скорости распространения любого возмущения в пористых средах.

Введение новой независимой переменной $v=u \cdot \exp (b y / 2)$ позволяет записать уравнение (8) и условие (9) в виде

$$
\begin{aligned}
\operatorname{sign} y|y|^{m} v_{x x}+v_{y y}-\left(b^{2} / 4\right) v & =f(x, y) \exp (b y / 2) ; \\
\frac{\partial}{\partial y} \exp \left(-\frac{b y}{2}\right) \int_{0}^{r} v(x, y) d x & =A|y|^{m} \operatorname{sign} y .
\end{aligned}
$$

В случае горизонтального непроницаемого водоупора $\left(k_{0}=0\right)$ в (8)-(11) можно положить $b=0$. Тогда условие (11) совпадает с (9), а уравнение (10) примет вид

$$
\operatorname{sign} y|y|^{m} \cdot v_{x x}+v_{y y}=f(x, y), 0<x<r .
$$

Нелокальная начально-краевая задача для уравнения Лаврентьева-Бицадзе.

Рассмотрим уравнение (12) в случае, когда $m=0, f(x, y)=0$ в прямоугольной области $\Omega_{n}=\{(x, y): 0<x<a,-n a<y<\beta\}$, где $a$ и $\beta$ - заданные положительные числа, $n=1,2,3 \ldots$

Уравнение Лаврентьева-Бицадзе

$$
\operatorname{sign} y \cdot u_{x x}+u_{y y}=0
$$

описывает динамику грунтовых вод, при отсутствии внешнего воздействия на поток.

Введем обозначения: $\Omega^{+}=\{(x, y): 0<x<a, 0<y<\beta\} \quad$ - эллиптическая, $\Omega_{n}^{-}=\{(x, y): 0<x<a,-n a<y<0\}$ - гиперболическая части области $\Omega_{n} ; \Delta_{1}$ - треугольная область, ограниченная отрезком $\overline{A B}: \quad 0 \leq x \leq a$ прямой $y=0$, характеристиками $A C: x+y=0$ и $B C: x-y=a$ уравнения (13); $\Omega=\Omega^{+} \cup \Delta_{1} \cup A B ; \bar{\Omega}_{n}$ - замыкание области $\Omega_{n}$.

В дальнейшем под решением $u(x, y)$ уравнения (13) в области $\Omega_{n}$ будем понимать такое решение, которое обладает следующими свойствами:

1) $u(x, y) \in C^{2}\left(\Omega^{+}\right) \cap C^{1}(\Omega) \cap C\left(\bar{\Omega}_{n}\right)$;

2) $u(x, y)$ в замыкании $\bar{\Omega}_{n}$ представимо в виде $u(x, y)=f_{1}(x-y)+f_{2}(x+y)$, где $f_{1}$ и $f_{2}-$ непрерывные функции.

В работе исследуется следующая смешанная задача $\mathrm{S}$.

$3 \boldsymbol{a} \boldsymbol{\partial} \boldsymbol{a} \boldsymbol{y} \boldsymbol{a} S$. Найти решение $u(x, y)$ уравнения (13), удовлетворяющее условиям

$$
\begin{gathered}
\frac{\partial}{\partial y} \int_{0}^{a} u(x, y) d x=\mu(y), 0<y<\beta ; \\
u(x,-n a)=h_{n}(x), u(x, b)=h(x), 0 \leq x \leq a ; \\
u(a, y)=\Phi_{a}(y),-a n \leq y \leq \beta ; \\
u(0, y)=\Phi_{0}(y),-a n \leq y \leq 0,
\end{gathered}
$$


где $\mu(y), \quad h_{n}(x), \quad h(x), \quad \Phi_{0}(y), \quad \Phi_{a}(y)$ - заданные функции, $\mu(y) \in C^{1}[0, \beta]$, $\Phi_{a}(y) \in C[-a n, \beta], \Phi_{0}(y) \in C[-a n, 0], h_{n}(x), h(x) \in C[0, a]$.

Краевые условия (15)-(17) являются локальными, а условие (14) представляет собой нелокальное условие типа условия А.А. Самарского.

Л е м м а 1 . Пусть существует решение $u(x, y)$ задачи S. Тогда для любой точки $x \in[0, a]$ справедливо

где

$$
\begin{gathered}
u(x, 0)=\sum_{k=1}^{n}\left\{\alpha_{k}\left[\Phi_{a}(x-k a)+\Phi_{0}(-x-(k-1) a)\right]-\right. \\
\left.-\alpha_{k+1}\left[\Phi_{a}(-x-(k-1) a)+\Phi_{0}(x-k a)\right]\right\}+H_{n}(x), \\
\alpha_{k}=\left\{\begin{array}{llll}
1, & k \equiv 1 & (\bmod 2) ; \\
0, & k \equiv 0 & (\bmod 2),
\end{array} H_{n}=\left\{\begin{array}{ccc}
-h_{n}(a-x), & n \equiv 1 \quad(\bmod 2) ; \\
h_{n}(x), & n \equiv 0 & (\bmod 2) .
\end{array}\right.\right.
\end{gathered}
$$

Доказательство леммы 1 можно провести методом математической индукции, опираясь на теорему о среднем значении для одномерного волнового уравнения.

С помощью леммы 1 и равенства $\frac{\partial}{\partial x}\left(u_{x}^{2}-u_{y}^{2}\right)+2 \frac{\partial}{\partial y}\left(u_{x} u_{y}\right)=0$, справедливого для любой гармонической в области $\Omega^{+}$функции $u(x, y)$, можно доказать следующую теорему единственности решения задачи $\mathrm{S}$.

T е о р е м а 1. Пусть $u(x, y)$ - решение однородной задачи, соответствующей задачи S, которое непрерывно дифференцируемо всюду в $\bar{\Omega}^{+}$, за исключением угловых точек границы $\Omega^{+}$, и обладает тем свойством, что $u_{x}(0, y), u_{x}(a, y) \in L^{2}(0, \beta)$. Тогда $u(x, y) \equiv 0$.

Решение смешанной задачи $\mathrm{S}$ будем искать в классе функций, обладающих тем свойством, что $u_{x x}(x, y) \in L[0, a]$ для любого $y \in(0, \beta)$.

Пусть $u=u(x, y)$ - решение задачи S. Тогда для любого $y \in(0, \beta)$ в силу условия (14) имеем

$$
\int_{0}^{a} u_{x x}(x, y) d x=-\frac{\partial^{2}}{\partial y^{2}} \int_{0}^{a} u(x, y) d x=-\mu^{\prime}(y) .
$$

Отсюда следует, что искомое решение удовлетворяет краевому условию с локальным смещением [1]:

$$
u_{x}(a, y)-u_{x}(0, y)=-\mu^{\prime}(y), 0<y<b .
$$

Через $\tau(x)$ обозначим правую часть равенства (18). Решение $u(x, y)$ задачи $\mathrm{S}$, согласно (15), (16) и (19), должно быть решением в области $\Omega^{+}$следующей краевой задачи для уравнения Лапласа:

$$
u_{x x}+u_{y y}=0 .
$$

3 a d а ч а $S_{1}$. Найти регулярное в области $\Omega^{+}$решение $u(x, y)$ уравнения (13) из класса $C\left(\bar{\Omega}^{+}\right)$, которое удовлетворяет условию гладкости $u_{x}(x, y) \in C(0 \leq x \leq a, 0 \leq y \leq b)$ и краевым условиям: $\quad u(x, 0)=\tau(x), \quad u(x, \beta)=h(x), \quad 0 \leq x \leq a ; \quad u(a, y)=\Phi_{a}(y)$, $u_{x}(a, y)-u_{x}(0, y)=\mu_{1}(y), 0<y<\beta$, где $\mu_{1}(y)=-\mu^{\prime}(y)$.

При достаточно гладких входных данных $\tau(x), h(x), \Phi_{a}(y)$ и $\mu_{1}(y)$ существование решения задачи $S_{1}$ доказывается методом редукции к интегральному уравнению Фредгольма второго рода, ядро которого однозначно определяется функцией Грина задачи Дирихле для уравнения (20) в области $\Omega^{+}$.

Решение задачи $\mathrm{S}$ в области $\bar{\Omega}_{n}^{-}$строится как гладкое продолжение решения задачи $\mathrm{S}_{1}$ из области $\Omega^{+}$в решение смешанной задачи для одномерного волнового уравнения.

Прикладные задачи, приводящие к задачам с нелокальным условием (14), требуют разработки приближенных методов решения этих задач. Поэтому мы остановимся на демонстрации 
метода Фурье решения задачи $\mathrm{S}_{1}$, ограничиваясь случаем, когда $a=\beta=1, h(x)=0$, $\Phi_{a}(y) \equiv 0, \mu_{1}(y) \equiv 0,0 \leq x \leq 1,0 \leq y \leq 1$.

Пусть требуется найти регулярное в области $\Omega^{+}=\{(x, y): 0<x<1,0<y<1\}$ решение $u(x, y)$ уравнения (20) из класса $C\left(\bar{\Omega}^{+}\right)$, такое, что $u_{x} \in C(0 \leq x \leq 1,0 \leq y<1)$ и

$$
\begin{gathered}
u(x, 0)=\tau(x), 0 \leq x \leq 1 ; \\
u(1, y)=0,0 \leq y \leq 1 ; \\
u_{x}(0, y)-u_{x}(1, y)=0,0<y<1 ; \\
u(x, 1)=0,0 \leq x \leq 1,
\end{gathered}
$$

где $\left.\tau(x) \in C[0,1] \cap C^{2}\right] 0,1[$.

Найдем класс нетривиальных решений задачи (21)-(24) для уравнения (20), представимых в виде

$$
u(x, y)=U(x) V(y) .
$$

Подставляя (25) в (20) и принимая во внимание (21)-(24), получим

$$
\begin{gathered}
U^{\prime \prime}(x)+\lambda U(x)=0,0<x<1 ; \\
U(1)=0 ; \\
U^{\prime}(0)=U^{\prime}(1) ; \\
V^{\prime \prime}(y)-\lambda V(y)=0 ; \\
V(1)=0 .
\end{gathered}
$$

В уравнении (26) введем новые независимые переменные:

$$
X(t)=U(x), x=1-t .
$$

Тогда в силу (26)-(28) получаем

$$
\begin{gathered}
X^{\prime \prime}(t)+\lambda X(t)=0, \quad 0 \leq t \leq 1 ; \\
X(0)=0, \quad X^{\prime}(0)=X^{\prime}(1) .
\end{gathered}
$$

Известно [2,3], что числа $\lambda_{k}=(2 \pi k)^{2}, k=0,1, \ldots$, и функции

$$
X_{0}(t)=t, X_{2 k-1}(t)=t \cos (2 \pi k t), X_{2 k}(t)=\sin (2 \pi k t)
$$

соответственно являются собственными значениями, собственными и присоединенными функциями задачи (32)-(33).

Следовательно, связь (31) позволяет утверждать, что система собственных и присоединенных функций задачи (26)-(28) задается следующим образом:

$$
U_{0}(x)=1-x, U_{2 k-1}(x)=(1-x) \cos (2 \pi k x), U_{2 k}(x)=-\sin (2 \pi k t) .
$$

Пусть $Y_{0}(t)=2, Y_{2 k-1}(t)=4 \cos (2 \pi k t), Y_{2 k}(t)=4(1-t) \sin (2 \pi k t), k=1,2, \ldots$ - система собственных и присоединенных функций задачи

$$
Y^{\prime \prime}(t)+\lambda Y(t)=0, Y(0)=Y(1), Y^{\prime}(1)=0 .
$$

Отсюда после замены $t=1-x$ получаем систему собственных и присоединенных функций: $v_{0}(x)=2, v_{2 k-1}(x)=4 \cos (2 \pi k x), v_{2 k}(x)=-4 x \sin (2 \pi k x), \kappa=1,2, \ldots$ задачи, сопряженной задаче (26) - (28).

По условию $\tau(x) \in C[0,1]$. Из базисной системы (34) следует, что функцию $\tau(x)$ можно разложить в биортогональный ряд [2, 3]

$$
\tau(x)=\tau_{0} U_{0}(x)+\sum_{k=1}^{\infty}\left[\tau_{2 k} U_{2 k}(x)+\tau_{2 k-1} U_{2 k-1}(x)\right],
$$

где $\tau_{0}=\left(\tau, \cup_{0}\right)_{0} ; \tau_{2 k}=\left(\tau, \mathcal{v}_{2 k}\right)_{0} ; \tau_{2 k-1}=\left(\tau, \cup_{2 k-1}\right)_{0}$. Здесь $(\cdot, \cdot)$ означает скалярное произведение в $L_{2}[0,1]$.

При $\lambda>0$ общее решение уравнения (29) имеет вид

$$
V(y)=C_{1} \operatorname{ch}(\sqrt{\lambda} y)+C_{2} \operatorname{sh}(\sqrt{\lambda} y),
$$

где $C_{1}$ и $C_{2}$ - произвольные постоянные.

Удовлетворив (36) условию (30) и дополнительному условию $V(0)=1$, получим 


$$
V(y)=\operatorname{sh}(\sqrt{\lambda} y)-\operatorname{cth}(\sqrt{\lambda}) \operatorname{ch}(\sqrt{\lambda} y) .
$$

Из (37) при $\lambda=\lambda_{k}=(2 \pi k)^{2}, \kappa=0,1, \ldots$ приходим к следующей системе функций, удовлетворяющих (29) и (30):

$$
V_{0}(y)=1-y, V_{k}(y)=\operatorname{sh}(2 \pi k y)-c \operatorname{th}(2 \pi k) \operatorname{ch}(2 \pi k y), \kappa=1,2, \ldots
$$

Решение задачи (21) - (24) для уравнения (20) будем искать в виде ряда

$$
u(x, y)=\tau_{0} U_{0}(x) V_{0}(y)+\sum_{k=1}^{\infty}\left[\tau_{2 k} U_{2 k}(x) V_{k}(y)+\tau_{2 k-1} v_{k}(x, y)\right],
$$

где $v_{k}(x, y)=U_{2 k-1}(x) V_{k}(y)-2 \sqrt{\lambda_{k}} U_{2 k}(x) z_{k}(y)$.

Непосредственным вычислением, как и в случае уравнения теплопроводности [2, 3], можно убедиться, что функция $u(x, y)$, задаваемая формулой (39), будет решением уравнения (20) и будет удовлетворять условиям (21), (22) тогда и только тогда, когда

$$
z_{k}(0)=0, z_{k}(1)=0, z_{k}^{\prime \prime}(y)-\lambda_{k} z_{k}(y)=-V_{k}(y) .
$$

Действительно, пусть $u_{k}(x, y)=\tau_{2 k} U_{2 k}(x) V_{k}(y)+\tau_{2 k-1} v_{k}(x, y)$. Легко видеть, что

$$
\begin{gathered}
\Delta u_{k}=\tau_{2 k}\left[U_{2 k}^{\prime \prime}(x) V_{k}(y)+U_{2 k}(x) V_{k}^{\prime \prime}(y)\right]+\tau_{2 k-1}\left[U_{2 k-1}^{\prime \prime}(x) V_{k}(y)-\right. \\
\left.-2 \sqrt{\lambda_{k}} U_{2 k}^{\prime \prime}(x) z_{k}(y)+U_{2 k-1}(x) V_{k}^{\prime \prime}(y)-2 \sqrt{\lambda_{k}} U_{2 k}(x) z_{k}^{\prime \prime}(y)\right] .
\end{gathered}
$$

Отсюда в силу свойств систем функций (34) и (38) получаем

$$
\begin{gathered}
\Delta u_{k}(x, y)=\tau_{2 k-1}\left[V_{k}\left(U_{2 k-1}^{\prime \prime}+\lambda_{k} U_{2 k-1}\right)-2 \sqrt{\lambda_{k}} U_{2 k}\left(z_{k}^{\prime \prime}-\lambda_{k} z_{k}\right)\right], \\
U_{2 k-1}^{\prime \prime}+\lambda_{k} U_{2 k-1}=-2 \sqrt{\lambda_{k}} U_{2 k} .
\end{gathered}
$$

Поэтому $\Delta u_{k}=-2 \tau_{2 k-1} \sqrt{\lambda_{k}} U_{2 k}\left[V_{k}+z_{k}^{\prime \prime}+\lambda_{k} z_{k}\right]=0$. Из (41) следует (40).

Поскольку функция $\left.\tau(x) \in C[0,1] \cap C^{2}\right] 0,1[$ и представима в виде (35), то легко видеть, что (39) удовлетворяет условиям (21)-(24). Из (39) имеем

$$
\lim _{y \rightarrow+0} u_{y}=v(x)=-\tau_{0} U_{0}(x)+\sum_{k=1}^{\infty}\left\{V_{k}^{\prime}(0)\left[\tau_{2 k} U_{2 k}(x)+\tau_{2 k-1} U_{2 k-1}(x)\right]-2 \sqrt{\lambda_{k}} \tau_{2 k-1} U_{2 k}(x) z_{k}^{\prime}(0)\right\} .
$$

После того как найдены $\tau(x), v(x)$, решение $u(x, y)$ задачи $\mathrm{S}$ в области $\Omega_{n}^{-}$определяется как решение смешанной задачи

$$
\begin{gathered}
u(x, 0)=\tau(x), \quad u_{y}(x, 0)=v(x), \quad 0<x<1 ; \\
u(0, y)=0, \quad u(a, y)=0, \quad-a n \leq y \leq 0
\end{gathered}
$$

для одномерного волнового уравнения

$$
u_{y y}=u_{x x} .
$$

В случае, когда $n=1$, функция $\tau(x)=-h_{1}(1-x)$ и решение задачи $\mathrm{S}$ в области $\Delta_{1}$ находится как решение задачи Коши (42) для уравнения (44). Оно имеет вид

$$
u(x, y)=\frac{-h_{1}(1-x-y)-h_{1}(1-x+y)}{2}+\frac{1}{2} \int_{x-y}^{x+y} v(t) d t
$$

Из (45) в силу (44), принимая во внимание, что $\tau(0)=-h_{1}(1)=0, \tau(1)=-h_{1}(0)=0$, легко видеть, что

где

$$
\begin{gathered}
u\left(\frac{x}{2},-\frac{x}{2}\right)=\Psi_{0}(x), u\left(\frac{1+x}{2}, \frac{x-1}{2}\right)=\Psi_{1}(x), 0 \leq x \leq 1, \\
2 \Psi_{0}(x)=-h_{1}(1-x)-\int_{0}^{x} v(t) d t, 2 \Psi_{1}(x)=-h_{1}(1-x)+\int_{1}^{x} v(t) d t .
\end{gathered}
$$

Решение задачи $\mathrm{S}$ в любой точке $(x, y) \in \Omega_{1}^{-}$, лежащей вне области $\Delta_{1}$, определяется с помощью формулы (46) и теоремы о среднем значении для уравнения (44). 


\section{БИБЛИОГРАФИЧЕСКИЙ СПИСОК}

1. Нахушев А.М. Уравнения математической биологии. М.: Высш. шк., 1995. 301 с.

2. Ионкин Н.И. Решение одной краевой задачи теории теплопроводности с неклассическим краевым условием // Дифференц. уравнения. 1977. Т. 13. № 2. С. 294-304.

3. Ионкин Н.И., Моисеев Е.И. О задаче для уравнения теплопроводности с двухточечными краевыми условиями // Дифференц. уравнения. 1979. Т. 15. № 7. С. 1284-1295.

Поступила 3.06.2003 г. 